\title{
ESTUDIO DEL EFECTO DEL POLVO Y ESTIMACIÓN DE LA POTENCIA NOMINAL EN UN STRING FOTOVOLTAICO
}

\author{
STUDY OF THE DUST EFFECT AND THE NOMINAL POWER \\ ESTIMATION IN A PHOTOVOLTAIC STRING

\section{José Angulo-Abanto ${ }^{1} \odot$, Brando Calsi-Silva ${ }^{1} \odot$, Erick Alfaro-Collazos ${ }^{2}{ }^{\circledR}$, Luis Conde-Mendoza' ${ }^{1}$, Emilio Muñoz-Cerón ${ }^{3}{ }^{\oplus}$, Rolf Grieseler ${ }^{1}{ }^{\circ}$, Jorge Guerra-Torres ${ }^{1} \oplus$, Jan Palomino-Töfflinger ${ }^{1 *} \oplus$, Rafael Espinoza-Paredes ${ }^{2}{ }^{\oplus}$, Juan De la Casa-Higueras ${ }^{3}$ (1)}

${ }^{1}$ Departamento de Ciencias, Sección de Física, Pontificia Universidad Católica del Perú, Lima, Perú

${ }^{2}$ Centro de Energías Renovables, Universidad Nacional de Ingeniería, Lima, Perú

${ }^{3}$ Grupo IDEA, Departamento de Electrónica, Universidad de Jaén, Jaén, España

Recibido (Recieved): 05/02/2020 Aceptado (Accepted): 05/03/2020

\section{RESUMEN}

La cantidad de polvo depositado en la superficie de un panel depende de los parámetros ambientales. Estos son de naturaleza aleatoria (ej. humedad, velocidad del viento y temperatura ambiente) lo que dificulta su modelamiento teórico. El presente trabajo describe el efecto del polvo calculando el factor de reducción ( $\eta_{\text {polvo }}$ ) y modelando su dependencia en el tiempo. Para lograr esto, se llevó una campaña experimental en tres strings tándem (a-Si / $\mu \mathrm{c}-\mathrm{Si}$ ) de $1.15 \mathrm{~kW}$ ubicados en la ciudad de Lima, dividida en dos periodos de tiempo. El primer periodo, del 15.07.2016 al 04.07.2017, los tres string se limpiaron dos veces semanalmente. La potencia nominal fue calculada para los días con cielo despejado. Se encontró que entre cada string existen ligeras diferencias o mismatch en la potencia nominal, lo que se consideró estimar un factor de corrección (k) con el propósito de reajustar la potencia de salida. En la segunda etapa del 05.07.2017 al 05.07.2018, se dejó que el polvo se deposite de manera natural, estudiando el efecto del polvo en la caída energética mediante el factor de reducción. Comprender este factor será de importancia para las operaciones de mantenimiento de los strings ubicados en ese entorno específico.

Palabras Clave: Clave: sistema fotovoltaico, deposición del polvo, factor de reducción

\section{ABSTRACT}

The amount of dust deposited on the surface of a panel depends on the environmental parameters. These are random (eg. humidity, wind speed and ambient temperature) which makes it difficult to model them theoretically. This paper describes the effect of dust by calculating the derating factor ( $\eta_{\text {_polvo }}$ ) and modeling its dependence over time. To achieve this, an experimental campaign was carried out in three tandem (a-Si / $\mu \mathrm{c}-\mathrm{Si}$ ) of $1.15 \mathrm{~kW}$ controlled in the city of Lima, divided into two periods of time. In the first period, from 07.15.2016 to 04.07.2017, the three strings were cleaned twice per week. The nominal power was calculated for days with clear skies conditions. It was found that between each string there are slight differences or mismatches in the nominal power, which is considered to estimate a correction factor $(k)$ in order to readjust the output power. In the second stage from 05.07.2017 to 05.07.2018, the powder was allowed to deposit naturally, studying the effect of the dust on the energy fall through the reduction factor. Understanding this factor will be of importance for the maintenance operations of the ropes determined in that specific environment.

Keywords: photovoltaic system, dust deposition, reduction factor

\footnotetext{
* Corresponding author.:

E-mail: japalominot@pucp.edu.pe
} 


\section{INTRODUCCIÓN}

El principal factor que reduce la eficiencia de un generador FV es la cantidad de polvo depositado en la superficie. Esto depende del grado de contaminación de la ciudad, la naturaleza del suelo, tipo de polvo y factores de instalación[1]. Se encontró en la referencia [2] que la deposición de polvo reduce el rendimiento en términos de potencia de salida hasta un $50 \%$ en un periodo de seis meses. En algunas regiones tropicales, mostró que el efecto auto limpieza por la lluvia es mucho menor que el coste total por operaciones de limpieza periódica[3]. Sin embargo, en regiones con climas áridos o desérticos, bajas lluvias y altas tasas de suciedad, hay un gran esfuerzo por optimizar y determinar la frecuencia de limpieza; ya que naturalmente (por falta de lluvia y por baja velocidad de viento) no se pueden autolimpiar.

Para comprender el efecto, se debe estudiar la interacción entre el polvo y la superficie del módulo FV. Se considera una superficie de vidrio totalmente limpia y el polvo se trata como una partícula suspendida en un medio. Esta partícula tiene energía cinética que genera un movimiento aleatorio o mejor conocido como movimiento browniano. Esta energía de la partícula depende directamente de la temperatura ambiental, velocidad del viento y densidad del medio que lo rodea. En un instante dado una partícula de polvo colisiona inelásticamente con la superficie, iniciándose la formación de la capa de polvo. En la fig. 1(a) se muestra el diagrama de fuerzas de las principales interacciones (partículasuperficie) que se pueden dar. Brevemente se describe:

Fuerza de arrastre, producida por velocidad del viento, representado por las flechas de rodamiento, desprendimiento y deslizamiento en la fig. 1(a), normalmente tiene un efecto positivo en los mecanismos de limpieza natural. A mayor diámetro, el aire tiene mayor área de contacto para extraer el polvo depositado. Se ha reportado que para partículas con tamaño de $10 \mu \mathrm{m}$ fueron removidas con velocidades de viento $25 \mathrm{~m} / \mathrm{s}$ y para partículas mayores a $50 \mu \mathrm{m}$ la velocidad de viento necesaria es menor aún [4]. Sin embargo, el aumento de la velocidad del viento puede tener un efecto negativo ya que puede traer mayor cantidad de partículas.

La fuerza de capilaridad $\left(F_{c}\right)$, está relacionada con los puentes de líquido que se forman entre la superficie y el polvo, se ha observado que un incremento de la humedad relativa de 40 a $80 \%$ lleva a un aumento en la adhesión del $80 \%$, lo que representaría casi el 98\% de la fuerza de adhesión [5]. En climas con altos valores de humedad relativa (ej. Lima) presenta un papel importante en la acumulación de polvo ya que asiste en los procesos de sedimentación.

La fuerza Van der Waals $\left(F_{v d w}\right)$ aparecen entre la interacción dipolar en dos superficies en contacto. La morfología de superficial y la composición química del polvo presentan un papel importante en la magnitud de la fuerza. Técnicas en análisis de materiales de microscopia electrónica y composición ayudan a entender este efecto[6]. Además, la fuerza electrostática, producto de la polarización de la partícula, puede favorecer o no a la deposición, si es atractiva o repulsiva.

Por la inclinación de los módulos, la fuerza de gravedad tiende a favorecer el desprendimiento de las partículas. Sin embargo, la acumulación de polvo en la parte más baja del panel puede ocasionar sombras parciales. En instalaciones con seguimiento se ha autolimpiado en un $50 \%$ comparado con un sistema fijo, durante un periodo de 6 meses [7]. En sistemas de concentración solar con seguimiento se reporta pérdidas del $19 \%$ en verano [8].

El diagrama de fuerzas presentado trata de explicar de manera general la interacción entre el polvo y la superficie del panel. En la fig. 1(b), se muestra el proceso de sedimentación de una capa de polvo con las sales del medio, esta capa es asistida por la humedad o formación de roció. Los problemas que puede generar esta capa es la perdida permanente de potencia, dado que generan puentes solidos entre las partículas y la superficie, lo que llevaría a la remoción manual o mecánica [9]. 

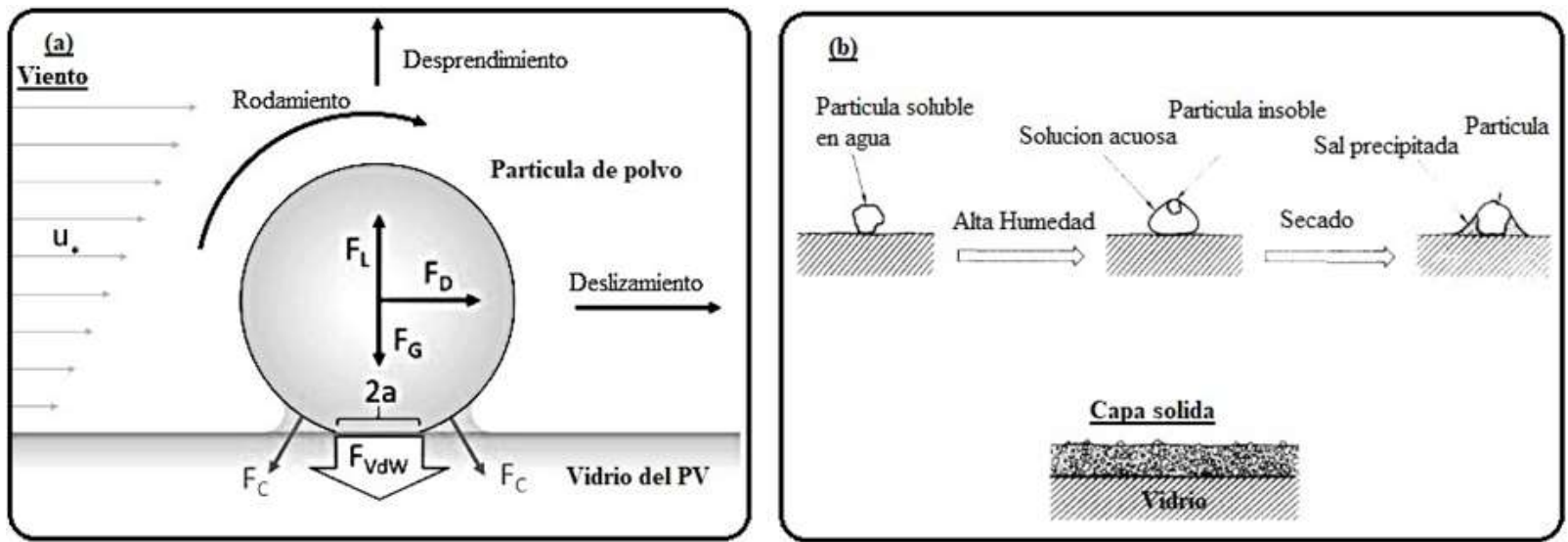

Fig. 1. Diagrama de fuerzas. (a) Esquema de las fuerzas que interactúan entre una partícula de polvo y la superficie de un vidrio [19], (b) Formación de una capa cementada de polvo [4].

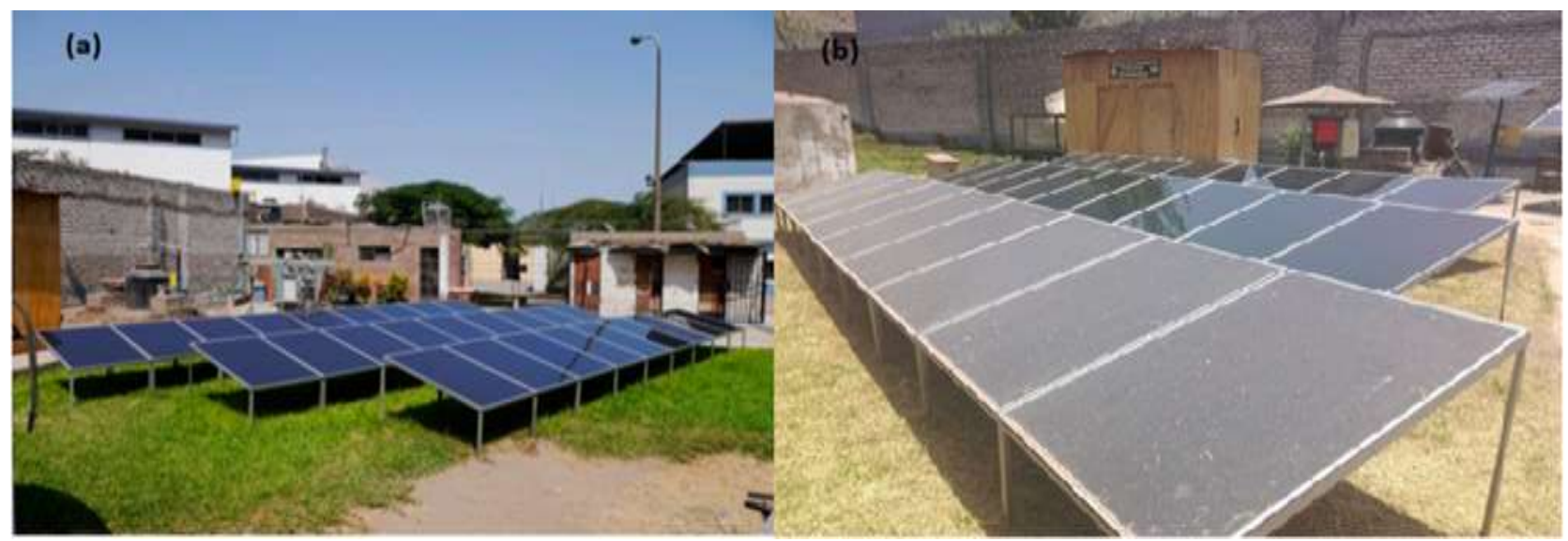

Fig. 2. Sistema FV de tecnología tándem. (a) Tres strings FV que se limpian semanalmente en el periodo de 15.07.2016 al 04.07.2017 [18], (b) Dos strings limpios y uno se deja que el polvo se deposita de manera natural en el periodo de 05.07.2017 al 05.07.2018.

Para medir el tamaño de partícula, morfología y composición involucra el uso de equipos en ciencia de materiales cómo es la microscopía electrónica de barrido, difracción de rayos $\mathrm{X}$, etc. Siendo estas medidas necesarias para comprender los mecanismos de adherencia y con ello establecer los pasos necesarios para la limpieza de estos vidrios. En la práctica, conviene medir los parámetros eléctricos (Potencia, voltaje y corriente) para determinar el grado de perdida en la eficiencia del sistema.

Finalmente, es importante mencionar que Lima es considerada, después del Cairo, la segunda ciudad más grande ubicada en un desierto [10] con precipitaciones de solo $9 \mathrm{~mm}$ por año. Esta cuidad cuenta con micro climas que dificultan la estimación de polvo depositado en un sistema FV. El trabajo pretende introducir un modelo lineal para estimar la ratio de deposición con el objetivo que puedan ser aplicados en las operaciones de mantenimiento. Nos enfocaremos en la estimación de la potencia nominal utilizando los datos experimentales correspondientes al primer año, así estudiar el estado del generador y luego modelar el efecto del polvo a través del factor de reducción

\section{CONFIGURACIÓN METODOLOGÍA}

El sistema FV de tecnología tándem (a-Si / $\mu \mathrm{c}-\mathrm{Si}$ ) implementado en el Centro de Energías Renovables de la Universidad Nacional de Ingeniería (CER-UNI) se utilizó para el estudio. Este sistema cuenta con tres strings con ficha técnica en condiciones estándar de medida, CEM, (irradiancia $1000 \mathrm{~W} / \mathrm{m}^{2}$, temperatura de célula $25^{\circ} \mathrm{C}$ y espectro AM 1,5G) mostradas en la tabla I. Las mediciones fueron durante un año (15.07.2016 al 04.07.2017) limpiadas dos veces por semana (fig. 2.a) y para el segundo año (05.07.2017 al 05.07.2018) se dejó que uno de ellos se deposite la suciedad de manera natural (fig. 2.b) sin limpieza. La potencia de salida $\left(P_{d c}\right)$, Irradiancia $(G)$ y temperatura del módulo $\left(T_{m}\right)$ fueron monitoreados cada 15 segundos. 
TABLA I

Especificaciones eléctricas del módulo tándem (a-Si / $\mu \mathrm{c}-\mathrm{Si}$ )

\begin{tabular}{lc}
\hline \multicolumn{1}{c}{ CARACTERÍSTICA } & Unidad \\
\hline Máxima Potencia & $128 \mathrm{~W}$ \\
Voltaje de Máxima Potencia & $45,4 \mathrm{~V}$ \\
Corriente de Máxima Potencia & $2,82 \mathrm{~A}$ \\
Voltaje de Circuito Abierto & $59,8 \mathrm{~V}$ \\
Corriente de Corto Circuito & $3,45 \mathrm{~A}$ \\
Coeficiente de temperatura para la potencia $\gamma$ & $-0,24 \%{ }^{\circ} \mathrm{C}$ \\
\hline
\end{tabular}

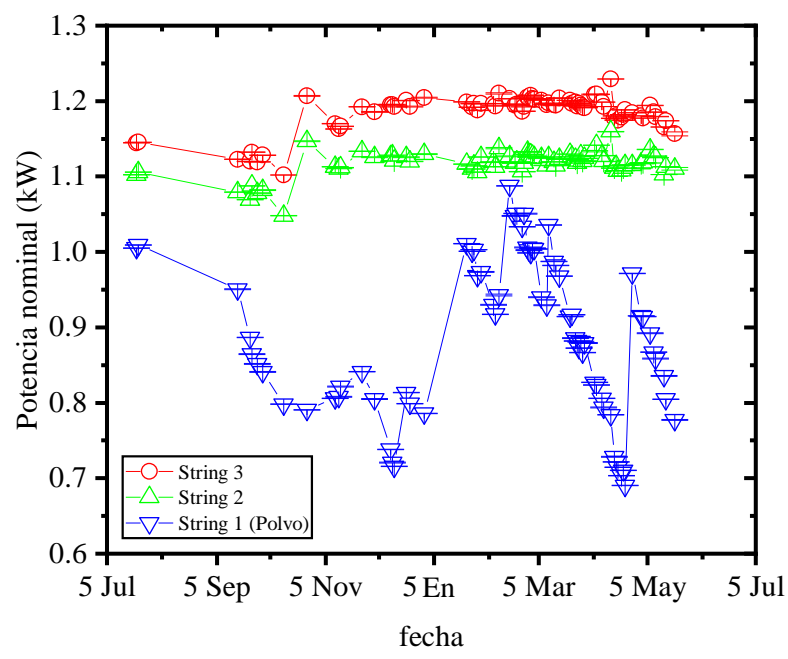

Fig. 1 Estimación de la potencia nominal para el periodo 05.07.2017 al 05.07.2018, depositándose polvo de manera natural.

\subsection{ESTIMACIÓN DE LA POTENCIA NOMINAL}

Para estimar la potencia nominal en el periodo limpio (15.07.2016 al 04.07.2017), los pasos sugerido en [11] fueron considerados. Es importante mencionar que se está obviando las recomendaciones instrumentales que sugiere este procedimiento. Sin embargo, es suficiente para hacer una evaluación del efecto del polvo y del sistema. Este procedimiento considera la ecuación empírica en [12] para estimar la potencia nominal. Esta ecuación (1) describe la relación entre la potencia de salida en el punto de máxima potencia $\left(P_{d c}\right)$ con las condiciones de operación, temperatura de modulo $\mathrm{T}_{\mathrm{m}}$ e irradiancia incidente en el plano $\mathrm{G}$.

$$
P_{D C}=P_{M}^{*} \times \frac{G}{G^{*}}\left(1+\gamma\left(T_{\mathrm{m}}-25^{\circ} \mathrm{C}\right)\right)
$$

Donde $G^{*}$ es la irradiancia a condiciones CEM, $\gamma$ coeficiente de temperatura y $P_{M}^{*}$ es la potencia nominal a CEM que se desea calcular. Dada la terna de valores monitorizados $\left(G, T_{m}, P_{d c}\right)$, el procedimiento [13] sugiere corregir en temperatura $\left(P_{D C} \rightarrow P_{\left(G, T \rightarrow 25^{\circ} \mathrm{C}\right)}\right)$, esto se logra evaluando en la ecuación (2).

$$
P_{\left(G, T \rightarrow 25^{\circ} \mathrm{C}\right)}=\frac{P_{\left(G, T_{\mathrm{m}}\right)}}{\left(1+\gamma\left(T_{\mathrm{m}}-25^{\circ} \mathrm{C}\right)\right)}
$$

Por último, para estimar la potencia nominal en condiciones reales de operación se realiza un ajuste lineal (3) en el rango de irradiancia de 800-1000 W/m²

$$
P_{\left(G, T \rightarrow 25^{\circ} \mathrm{C}\right)}=P_{M}^{*} \times \frac{G}{G^{*}}
$$

En el primer año de monitoreo, se observó entre los tres strings tienen ligeras variaciones en los valores absolutos de $P_{M}^{*}$. Esto está bien justificado en la literatura, ya que en un mismo lote de producción los módulos individuales pueden tener ligeras diferencias o variaciones en las eficiencias [14]. En el segundo año de estudio se observa la disminución en la potencia nominal por efecto de la deposición de polvo (fig.3). Decreciendo en torno al $30 \%$ para un tiempo de exposición de dos meses. La determinación de las pérdidas se evaluará más adelante con el factor de reducción.

\subsection{FACTOR DE REDUCCIÓN, $\eta_{\text {polvo }}$}

La potencia nominal calculada nos indica el estado general de cada string, pero no ayuda a determinar el efecto del polvo. Para ello, debemos identificar un factor de reducción que represente la perdida en la potencia de salida. De acuerdo a [15] reescribimos la ecuación 1:

$$
P_{D C}=P_{M}^{*} \times \eta \times \frac{G}{G^{*}}\left(1+\gamma\left(T_{\mathrm{m}}-25^{\circ} \mathrm{C}\right)\right)
$$

Donde $\eta=\eta_{\text {tiempo }} \eta_{\text {sombra }} \eta_{\text {polvo }}$ es el factor de reducción que depende a su vez de la degradación por el tiempo, sombras y polvo. El factor de reducción $\eta_{\text {polvo }}$ será de interés para el modelamiento del polvo. En la ecuación (5) se define el cálculo en función a la energía que entrega durante un día.

$$
\begin{gathered}
\eta_{\text {polvo }}=\frac{E_{\text {polvo }}}{E_{\text {limpio }}} ; \\
E_{i}=\int_{t_{\text {inicial }}}^{t_{\text {inicial }}+\Delta t} P_{d c} d t
\end{gathered}
$$

Donde $E$ es la energía que entrega el sistema en un intervalo de tiempo dado. Para evitar los efectos de sombras se tiene en cuenta irradiancias medidas mayores a $800 \mathrm{w} / \mathrm{m}^{2}$. En la fig. 4(a) se muestra la región considerada. Se observa que en las mismas condiciones de operación, la potencia de salida presenta ligeras diferencias. Para normalizar los tres strings, el factor de corrección $\left(K=E_{i} / E_{3}\right)$ es calculado para el string 1 y 2 . En la fig. 4(b), los valores de $K$ se muestra para cada día con cielo despejado. En el cuadro superior del gráfico, se presenta la distribución normal que tiene este parámetro. La 
media y su desviación estándar fueron calculados $\left(K_{1}=0.982 \pm 0.016\right.$ y $\left.K_{2}=0.943 \pm 0.015\right)$.

Los valores del factor de corrección están dentro de lo esperado [15]. Teniendo en cuenta que el string 1 es el que deposita el polvo y la corrección entre los strings, se calcula de la ecuación (5) el factor de reducción por efecto del polvo.

\section{ANÁLISIS DE RESULTADOS}

El factor de reducción indica que las perdidas pueden llegar hasta un 40 \% en la ubicación geográfica de la Universidad Nacional de Ingeniería, Lima-Perú. [16] demuestran que en cámaras controladas existe una relación lineal entre la densidad de polvo acumulado y la perdida de la potencia normalizada (1.7\% por $\left.\mathrm{g} / \mathrm{m}^{2}\right)$. En la fig. 5(a), es evidente que las perdidas presentan un comportamiento lineal con los días de exposición en algunos rangos de tiempo (ej. cuadrado sombreado en fig. 5(a)). Se han identificado seis regiones lineales de interés, etiquetadas con R1R6.

Los incrementos que se muestran en el factor de reducción, son atribuidos a los procesos de autolimpieza, como se mencionó en la introducción, producidos tal vez por un aumento en la velocidad del viento, lo que desprendería algunas partículas de polvo por aumento de la fuerza de arrastre. En general durante todo el año de estudio el factor de reducción tiene un comportamiento aleatorio ya que depende de las condiciones meteorológicas

En la fig. 5(b), se presenta la pendiente en los seis rangos de tiempo donde el factor de reducción tiene una caída lineal. En R1, se puede atribuir a la formación de la primera capa fina de polvo en la superficie del vidrio [17]. Las primeras interacciones se dan entre la superficie de vidrio limpio y las partículas suspendidas en el medio (ver fig. 1(a)) presentadas en la introducción
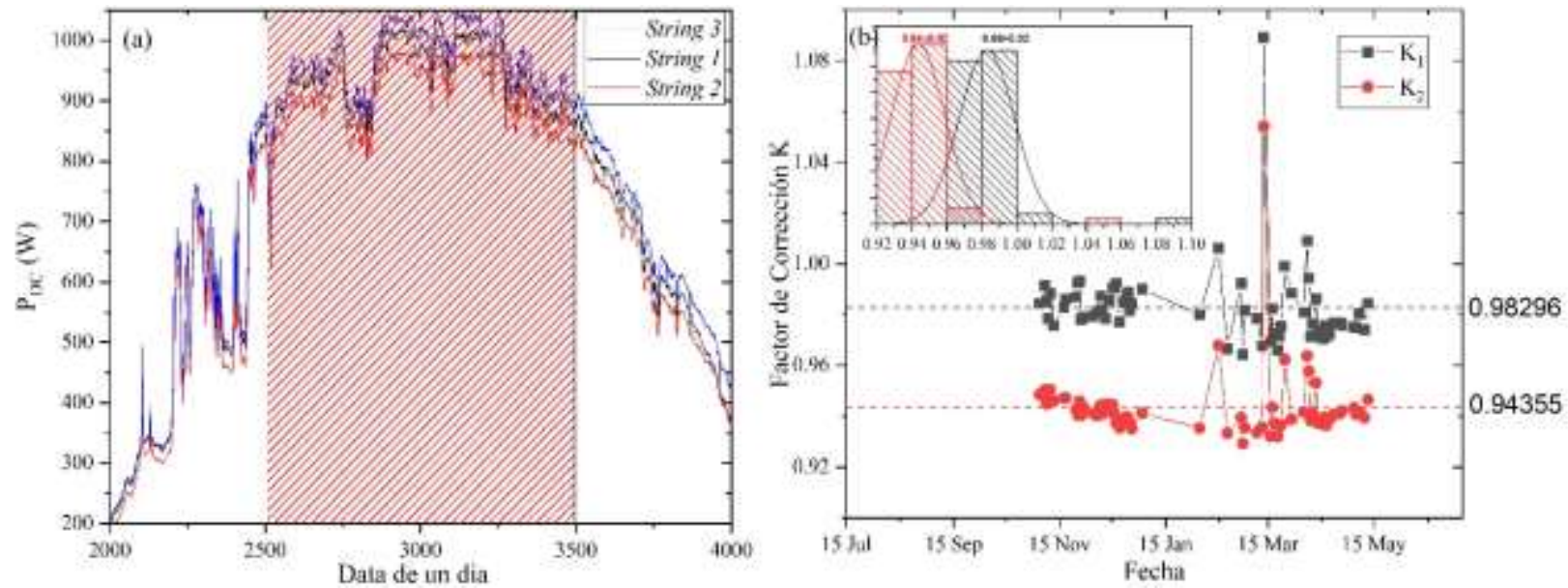

Fig. 4. (a) Ejemplo de la diferencia en la Potencia de salida de los tres strings para un día. (b) Cálculo de factor de corrección.
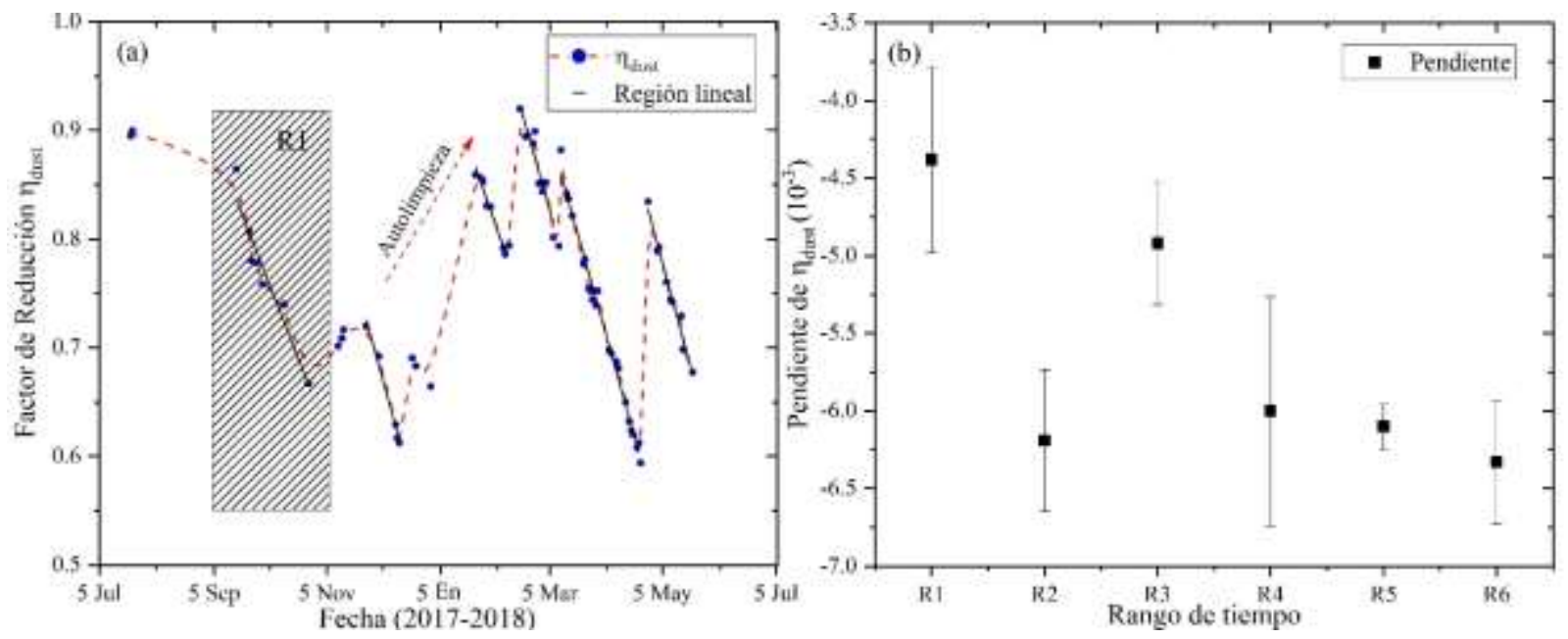

Fig. 5. Factor de reducción. (a) Cálculo del factor de reducción en el segundo año, el cuadrado sombreado con rayas muestra un ejemplo de los rangos de tiempo considerados, (b) se presenta las pendientes ajustadas en los seis rangos de tiempo considerados en (a). 
Las condiciones climáticas de Lima; por un lado, con alta humedad favorecerían a la formación de puentes húmedos, que apoyaría a la fijación de las partículas de polvo. Por otro lado, la fuerza de arrastre seria mucho menor que la fuerza de capilaridad debido a las bajas velocidades de viento, formando una capa fina de polvo en la superficie. En el caso R2, se puede inferir la aglomeración en la superficie del panel. Para $\mathrm{R}_{3}$, se puede atribuir a un proceso de autolimpieza, tal vez por un aumento en el volumen de las precipitaciones que se reporta en los meses de diciembre o enero.

Para R4-R6, se observa que la pendiente es casi constante, ya que la capa fina de polvo se ha formado una vez más y los procesos de deposición y desprendimiento de polvo se dan periódicamente.

A pesar que las pérdidas de la eficiencia por efecto de polvo es un fenómeno totalmente estocástico debido a que depende de factores ambientales, su modelamiento es muy complejo e impredecible. No obstante, los datos indican que cuando empieza a formarse la capa de polvo la perdida en la energía entregada respecto al panel limpio, presenta un comportamiento lineal.

\section{CONCLUSIONES}

- En Lima, la acumulación de polvo es uno de los principales factores que causa pérdidas en la eficiencia del sistema FV. La caracterización de este efecto debe ser realizado en la ubicación geográfica de la instalación ya que existen factores ambientales que no se pueden estimar ni predecir (ej. velocidad de viento, humedad relativa, grado de contaminantes en el aire, etc.).

- El cálculo de la potencia nominal, en el primer periodo de estudio, indica que el sistema tiene un comportamiento esperado, de acuerdo a las especificaciones de fabricante. Se calculó el factor de corrección K para luego ser implementado en el estudio del polvo.

- En el segundo periodo de evaluación, el polvo depositado presenta un papel importante en las pedidas energéticas que en promedio representan el $25 \%$ y mínima del $40 \%$. En la literatura, el factor de reducción es modelado con la densidad de polvo depositado en la superficie $(\mu \mathrm{g} / \mathrm{m} 2)$. Sin embargo, es evidente la dependencia lineal en el tiempo de exposición, lo que facilita la caracterización. Los rangos de tiempo estudiados indican la formación de una capa sedimentada. Se requiere una limpieza mecánica o manual para regresar al estado inicial del string.

- Finalmente, para lograr comprender mejor los mecanismos y el efecto que tiene el polvo se deben realizar medidas con microscopia electrónica, con el fin de tener información del tamaño de grano, morfología superficial, composición de la capa y degradación de los materiales. Además, medidas ópticas con equipos de transmisión de luz para calcular la absorbancia del polvo.

\section{AGRADECIMIENTOS}

Se agradece al Grupo IDEA de la Universidad de Jaén, España, por brindar el soporte científico realizado en la pasantía 'Movilización en Ciencia, Tecnología e Innovación tecnológica-Pasantías' bajo el contrato Nro. 161-2019-FONDECYT. Agradecimiento a la colaboración entre el Centro de Energías Renovables, Universidad Nacional de Ingeniería de Lima y el grupo IDEA por los datos proporcionados. Parte de este trabajo recibió apoyo financiero por el Fondo Nacional de Desarrollo Científico, Tecnológico y de Innovación Tecnológica (FONDECYT) mediante el proyecto con el contrato $\mathrm{N}^{\circ}$ 045-2018-FONDECYT-BM-IADT-MU.

\section{REFERENCIAS}

[1] M. R. Maghami, H. Hizam, C. Gomes, M. A. Radzi, M. I. Rezadad, and S. Hajighorbani, "Power loss due to soiling on solar panel: A review," Renew. Sustain. Energy Rev., vol. 59, pp. 1307-1316, 2016.

[2] M. Abderrezek and M. Fathi, "Experimental study of the dust effect on photovoltaic panels' energy yield," Sol. Energy, vol. 142, pp. 308-320, 2017.

[3] J. Tanesab, D. Parlevliet, J. Whale, and T. Urmee, "Dust Effect and its Economic Analysis on PV Modules Deployed in a Temperate Climate Zone," Energy Procedia, vol. 100, pp. 65-68, 2016.

[4] E. F. Cuddihy, "Theoretical Considerations of Soil Retention," Sol.Energy Mater., vol. 3, pp. 21-33, 1980.

[5] E. Y. T. Chen, L. Ma, Y. Yue, B. Guo, and H. Liang, "Measurement of dust sweeping force for cleaning solar panels," Sol. Energy Mater. Sol. Cells, vol. 179, pp. 247-253, 2018.

[6]W. Javed, Y. Wubulikasimu, B. Figgis, and B. Guo, "Characterization of dust accumulated on photovoltaic panels in Doha, Qatar," Sol. Energy, vol. 142, pp. 123-135, 2017.

[7] M. Adinoyi and S. Said, "Effect of dust accumulation on the power outputs of solar photovoltaic modules," Renew. Energy, vol. 60, pp. 633-636, 2013.

[8] J. Montes-Romero et al., "Impact of soiling on the outdoor performance of CPV modules in Spain," 15th Int. Conf. Conc. Photovolt. Syst., vol. 2149, p. 060004, 2019.

[9] H. A. Kazem and M. T. Chaichan, "The effect of dust accumulation and cleaning methods on PV panels' outcomes based on an experimental study of six locations in Northern Oman," Sol. Energy, vol. 187, pp. 30-38, 2019.

[10] M. Hussein, "Sustainable Regeneration Of Urban Green Areas In Egypt' S Desert Cities Sustainable Regeneration Of Urban Green Areas In Egypt's Desert Cities Adopting Green Infrastructure Strategies In New Borg El-Arab City," HafenCity University, 2018.

[11] F. Martínez-Moreno, E. Lorenzo, J. Muñoz, and R. Moretón, "On the testing of large PV arrays," Prog. Photovoltaics Res. Appl., vol. 20, no. 1, pp. 100-105, 2012.

[12] C. R. Osterwald, "Translation of device performance measurements to reference conditions," Sol. Cells, vol. 18, no. 3-4, pp. 269-279, 1986.

[13] F. Martinez, "Caracterizacion y Modelado de Grandes Centrales Fotovoltaicas," tesis doctoral, Escuela Politécnica de Madrid, Universidad Politécnica de Madrid, Madrid, MAD, 2012. 
[14] I. De la Parra, M. Muñoz, E. Lorenzo, M. García, J. Marcos, and F. Martínez-Moreno, "PV performance modelling: A review in the light of quality assurance for large PV plants," Renew. Sustain. Energy Rev., vol. 78, no. November 2016, pp. 780-797, 2017.

[15] M. Jaszczur et al., "The field experiments and model of the natural dust deposition effects on photovoltaic module efficiency," Environ. Sci. Pollut. Res., vol. 26, no. 9, pp. 8402-8417, 2019.

[16] A. A. Hachicha, I. Al-Sawafta, and Z. Said, "Impact of dust on the performance of solar photovoltaic (PV) systems under United Arab Emirates weather conditions," Renew. Energy, vol. 141, pp. 287-297, 2019.

[17] V. Gupta, M. Sharma, R. Kumar, and K. N. D. Babu, "Comprehensive review on effect of dust on solar photovoltaic system and mitigation techniques," Sol. Energy, vol. 191, pp. 596-622, 2019.

[18] I. Romero-Fiances, E. Muñoz-Cerón, R. Espinoza-Paredes, G. Nofuentes, and J. De La Casa, "Analysis of the performance of various pv module technologies in Peru," Energies, vol. 12, no. 1, 2019. [19] K. K. Ilse, B. W. Figgis, V. Naumann, C. Hagendorf, and J. Bagdahn, "Fundamentals of soiling processes on photovoltaic modules," Renew. Sustain. Energy Rev., vol. 98, pp. 239-254, 2018.

\section{(c) (1)}

Los artículos publicados por TECNIA pueden ser compartidos a través de la licencia Creative Commons: CC BY 4.0. Permisos lejos de este alcance pueden ser consultados a través del correo revistas@uni.edu.pe 